SIMULATION OF SEMICONDUCTOR PROCESSES AND DEVICES Vol. 12

Edited by T. Grasser and S. Selberherr - September 2007

\title{
Nonlinear Piezoresistance Effect in Devices with Stressed Etch Stop Liner
}

\author{
K.H. Bach, R. Liebmann, M. Nawaz, C. Jungemann*, E. Ungersboeck ${ }^{* *}$ \\ Infineon Technologies, Am Campeon 1-12, D-81726 Munich, Germany \\ Karl-Heinz.Bach@infineon.com,Rainer.Liebmann@infineon.com \\ Muhammad.Nawaz@infineon.com \\ *EIT4, Bundeswehr University, D-85577 Neubiberg, Germany \\ Christoph.Jungemann@unibw.de \\ ** Institute for Microelectronics, TU Wien, A-1040 Wien, Austria \\ Ungersboeck@iue.tuwien.ac.at
}

\begin{abstract}
Stressed etch stop liners (ESL) are a common way to increase device performance. Here we investigate the layout dependent channel stress for mono- and multi-layer deposition. By means of empirical pseudopotential method full band structures are calculated and based on full band Boltzmann equation mobilities are extracted. We present for the first time nonlinear mobility enhancement maps for two strain components, in channel and out-of-plane direction, showing that for typical ESL conditions both strain components are important for NFET and PFET.
\end{abstract}

\section{Stress Simulation}

First we investigate simulated deposition of an intrinsically stressed etch stop liner (ESL) by mono- and multilayer deposition: The latter method is known to produce larger stresses [1-2]. The layout dependence of channel stress is investigated by $3 \mathrm{D}$ simulations.

For a typical device (Fig. 1) we first show in Fig. 2 results for the width-averaged [3] Sxx at the center of the channel, depending on the distance poly-to-contact for 1 and 5 sublayers. The multi-layer case (red squares) shows larger stress and a stronger stress reduction for small poly-to-contact distance. Fig 3 shows corresponding curves for Syy (note the opposite sign here).

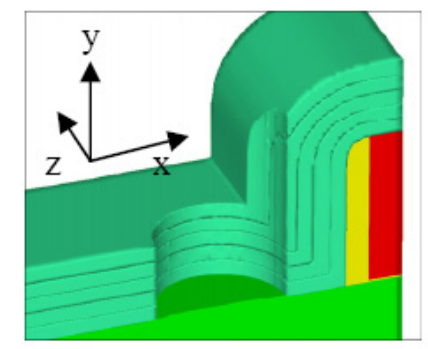

Figure 1: 3D structure of half device with contact hole and etch stop liner with 5 layers. 


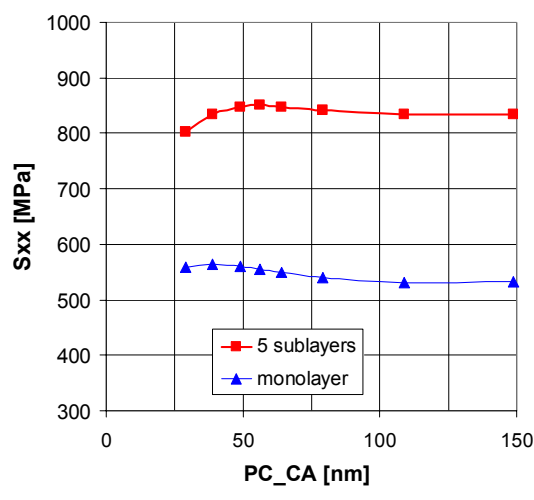

Figure 2: $\operatorname{Sxx}$ variation with distance poly-to-contact. Triangles: for monolayer case, squares: for 5 sublayer case.

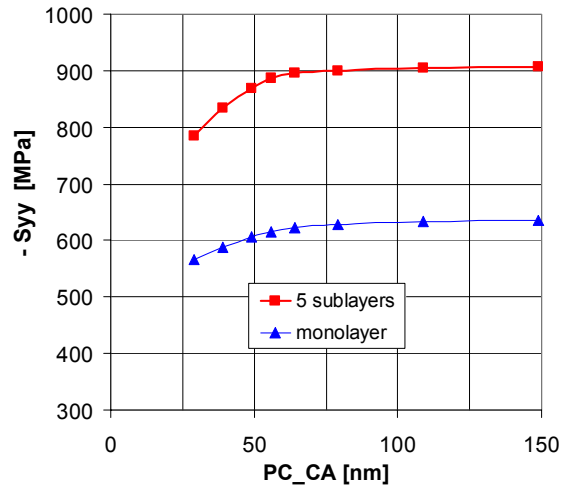

Figure 3: Same as Fig. 2, for -Syy. (Opposite sign as Sxx).

\section{Electrical Simulation}

For electrical simulations full band structures are calculated by means of empirical pseudopotential method (EPM) [4], then mobilities are extracted based on the full band Boltzmann equation. We have investigated bulk mobilities beyond the linear piezoresistance regime [5] by screening a large array of strain components in channel (Exx) and out-of-plane direction (Eyy), both ranging from $-1 \%$ to $+1 \%$. These components typically occur for ESL stress. We neglect Ezz which is zero for wide devices (plane strain condition). For all cases we calculated the band structure with EPM and extracted the bulk mobilities for $<110>$ and $<100>$ channel orientations.

\subsection{Electron mobilities}

Fig. 4 shows a contour plot of the electron bulk mobility enhancement for undoped silicon compared to the unstressed case. For both orientations the maximum is in the lower right corner where stress is tensile in channel direction, and compressive in outof-plane direction. A cut along the diagonal (Fig. 5) shows at low strain perfect agreement with the linear model, and at high strain saturation for both orientations. 
Edited by T. Grasser and S. Selberherr - September 2007
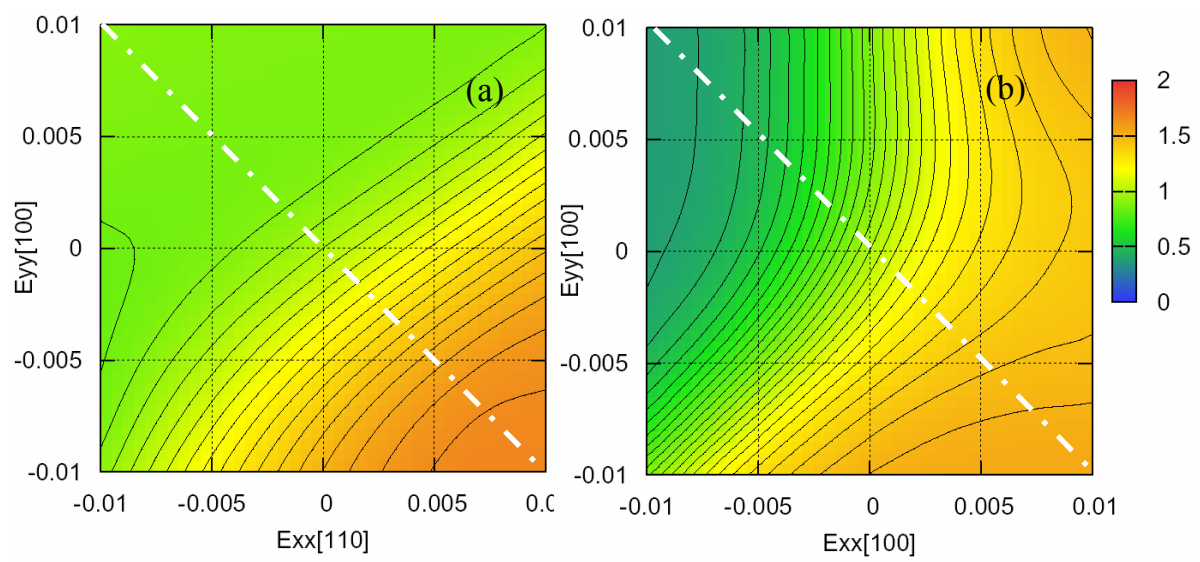

Figure 4: Enhancement map of electron bulk mobilities in undoped silicon as function of strain components Exx and Eyy, (a) for $<110>$ channel and (b) for $<100>$ channel. Ezz=0 assumed.
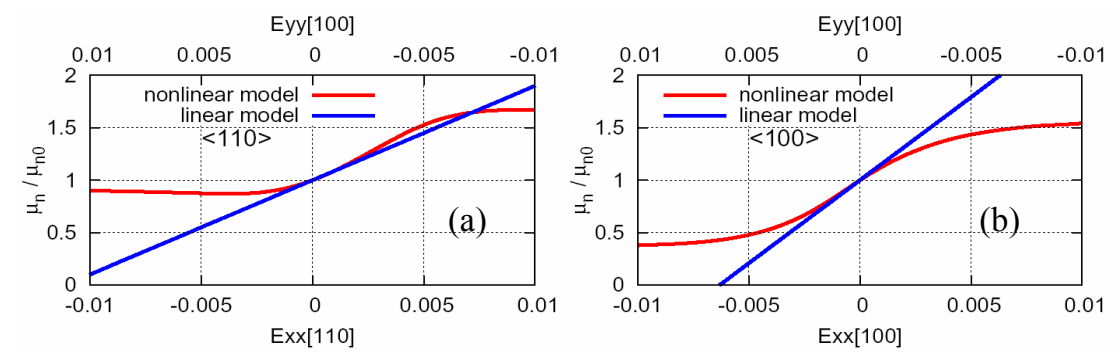

Figure 5: Enhancement of bulk electron mobilities along diagonal cuts in Fig. 4 compared to linear model.

\subsection{Hole mobilities}

Fig. 6 shows a contour plot of the hole bulk mobility enhancement for undoped silicon. For the $<110>$ channel the maximum is in the upper left corner where stress is compressive in channel direction, and tensile out-of-plane (exactly opposite to electrons), whereas for $\langle 100>$ a minimum is near the unstrained case, explaining why the linear model predicts such a low stress sensitivity. The cut along the diagonal (Fig. 7) for low strain again shows excellent agreement with the linear model. For high $<110>$ strain mobility enhancements above 5 can be achieved for undoped silicon.

\section{Conclusion}

Stress dependent bulk mobilities have been calculated for a large array of different strain conditions in channel- and out-of-plane-direction and the range relevant for ESL stress has been determined. The out-of-plane stress is found to be significant and has a strong impact on mobility. We have shown the nonlinear piezoresistance model 
to be in excellent agreement with the linear model [5] at low stress for both, electrons and holes. These findings significantly improve the understanding of the stress dependence of device performance.
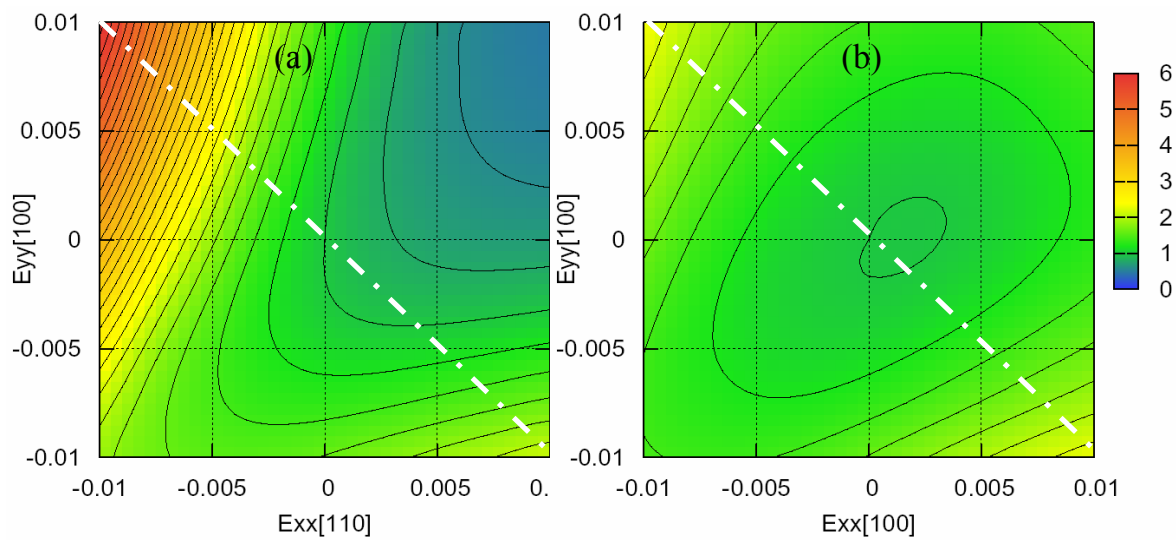

Figure 6: Enhancement map of hole bulk mobilities in undoped silicon as function of strain components Exx and Eyy, (a) for $<110>$ channel and (b) for $<100>$ channel. Ezz $=0$ assumed.
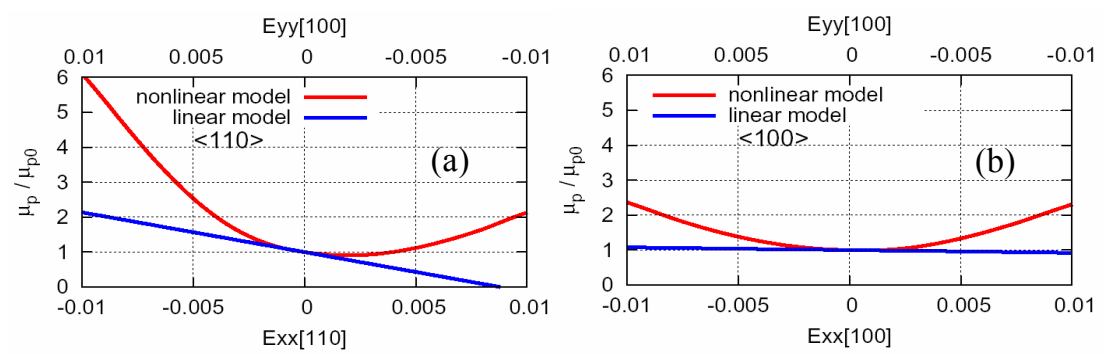

Figure 7: Enhancement of bulk hole mobilities along diagonal cut in Fig. 6 compared to linear model.

\section{References}

[1] K. Goto et al., "Technology booster using strain-enhancing laminated SiN (SELS) for 65nm node HP MPUs", IEDM Tech. Dig., pp. 209-212, December 2004.

[2] K. V. Loiko et al., "Multi-layer model for stressor film deposition", SISPAD 06, session 6.2, Sept. 2006.

[3] R. Liebmann, M. Nawaz, K. H. Bach, "Efficient 2D approximation for layout-dependent relaxation of etch stop liner stress due to contact holes", SISPAD 06, poster session, Sept. 2006.

[4] E. Ungersboeck, S. Dhar, G. Karlowatz, H. Kosina, S. Selberherr, "Physical modeling of electron mobility enhancement for arbitrarily strained silicon", J. Comput. Electronics, vol. 6 , pp. 55-58, 2007.

[5] C. S. Smith, "Piezoresistance effect in germanium and silicon", Phys. Rev., vol. 94, pp. 42-49, 1954. 